\title{
Achalasia cardia
}

\section{Praveer Rai • Sunilbaran Das Chakraborty}

Published online: 7 August 2012

(C) Indian Society of Gastroenterology 2012

A 44-year-old man presented with dysphagia to liquids and solids for 15 years, intermittent cough with expectoration for 1 year and four episodes of hematemesis. For the cough, he was suspected to have tuberculosis, and received antitubercular treatment with no response. He took analgesics for pain in knee joints infrequently. The patient had good appetite and no weight loss. He had pallor; no other significant findings were reported on clinical examination, including respiratory system.

$\mathrm{X}$-ray chest revealed a dilated esophagus. An upper gastrointestinal endoscopy showed a grossly dilated esophagus with food residue; the stomach could not be entered. Computerized tomography (Fig. 1) revealed a massively dilated esophagus occupying right side of the chest.

The diagnosis was confirmed by esophageal manometry. The manometry catheter could not be passed into stomach; all recording ports, placed in esophageal body, showed non-peristaltic simultaneous contraction. The patient underwent surgery.

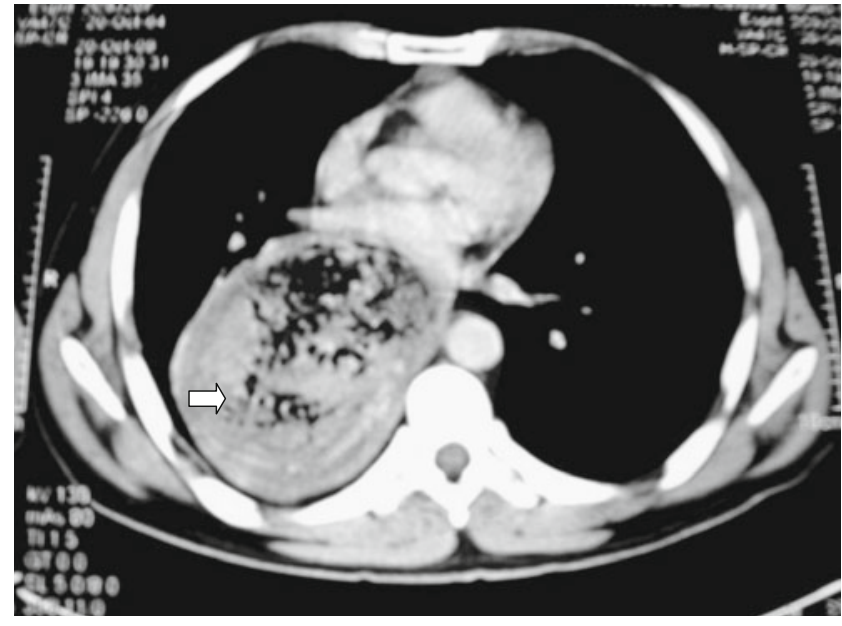

Fig. 1 CT scan of thorax showing dilated esophagus filled with food material (arrow)

P. Rai $(\bowtie) \cdot$ S. D. Chakraborty

Department of Gastroenterology,

Sanjay Gandhi Postgraduate Institute of Medical Sciences,

Raebareli Road,

Lucknow 226 014, India

e-mail: praveer_rai@yahoo.com 